\title{
DESCRIPTIVE SET THEORETIC METHODS APPLIED TO STRICTLY SINGULAR AND STRICTLY COSINGULAR OPERATORS
}

\author{
GEORGE ANDROULAKIS AND KEVIN BEANLAND
}

\begin{abstract}
The class of strictly singular operators originating from the dual of a separable Banach space is written as an increasing union of $\omega_{1}$ subclasses which are defined using the Schreier sets. A question of J. Diestel, of whether a similar result can be stated for strictly cosingular operators, is studied.
\end{abstract}

\section{INTRODUCTION}

The main results of this paper use descriptive set theory to demonstrate that a class of operators can be written as a union of $\omega_{1}$ many subclasses or as an intersection of $\omega_{1}$ many superclasses. The Schreier classes $\left(S_{\xi}\right)_{\xi<\omega_{1}}$ which were introduced by D. Alspach and S.A. Argyros ([1]) play an important role in defining these subclasses or superclasses. Other instances where certain notions where quantified by using the Schreier families $\left(S_{\xi}\right)_{\xi<\omega_{1}}$, are the $S_{\xi^{-}}$ unconditional basic sequences ([6], [13]), $S_{\xi}$-convex combinations of sequences in Banach spaces ([3, page 1054]), $S_{\xi}$-spreading models ([3, page 1057]), $\xi$-convergent sequences in Banach spaces ([3, page 1054]), $\xi$-Dunford-Pettis property ([3, page 1059]), $S_{\xi}$ strictly singular operators and $S_{\xi}$-hereditarily indecomposable Banach spaces ([2]).

There are two main parts in this article. The first is an extension of a result on strictly singular operators by the first named author, P. Dodos, G. Sirotkin and V. Troitsky, ([2]). The classes of $S_{\xi}$ strictly singular operators are introduced in [2] for $1 \leq \xi<\omega_{1}$. These are increasing subclasses of the class of strictly singular operators between two fixed Banach spaces. It is proved in [2] that the class of strictly singular operators between two fixed separable Banach spaces is equal to the union (for $1 \leq \xi<\omega_{1}$ ) of the classes of $S_{\xi}$ strictly singular operators between these spaces (Theorem 2.2). The first main result of the present article states that the same holds if we merely assume that the domain space has a separable predual (Theorem 2.3).

The second main part of this article (section 3) gives a partial answer to a question of J. Diestel [5]. The question asks whether the class of strictly cosingular operators between two fixed Banach spaces can be quantified by using the Schreier families $\left(S_{\xi}\right)_{\xi<\omega_{1}}$ and whether the new defined classes can be used to retrieve the class of strictly cosingular operators. In other

1991 Mathematics Subject Classification. 47B07, 47A15.

Key words and phrases. Strictly singular and cosingular operators, Schreier families, descriptive set theory. 
words, the question asks whether there exists a result for strictly cosingular operators which is similar to Theorems 2.2 and 2.3. We answer this question under additional assumptions on the range space (Theorem 3.1). The assumptions on the range space can be reduced if we work with a new class of operators whose definition is similar to the definition of strictly cosingular operators: Given a Banach space $Y$ and a family $\left(\mathcal{A}_{i}\right)_{i \in I}$ of normalized basic sequences of the dual space $Y^{*}$, we define the notion of $\left(\mathcal{A}_{i}\right)_{i \in I}$ strictly cosingular operators from a Banach space $X$ to $Y$ (their class is denoted by $\left(\mathcal{A}_{i}\right)_{i \in I}-\mathcal{S C}(X, Y)$ ). These are defined in a similar manner to strictly cosingular operators, and for some choices of $\left(\mathcal{A}_{i}\right)_{i \in I}$, they coincide with the class of strictly cosingular operators. Also for $1 \leq \xi<\omega_{1}$ we define the class of $\left(\mathcal{A}_{i}\right)_{i \in I^{-}} S_{\xi}$ strictly cosingular operators from $X$ to $Y$ (their class is denoted by $\left(\mathcal{A}_{i}\right)_{i \in I^{-}} \mathcal{S C}_{\xi}(X, Y)$ ). These are decreasing classes of operators containing the class $\left(\mathcal{A}_{i}\right)_{i \in I^{-}} \mathcal{S C}(X, Y)$. We prove that if $Y$ is separable and $\mathcal{A}_{i}$ is analytic for all $i \in I$ then the class $\left(\mathcal{A}_{i}\right)_{i \in I^{-}} \mathcal{S C}(X, Y)$ is equal to the intersection of $\left(\mathcal{A}_{i}\right)_{i \in I^{-}} \mathcal{S C}_{\xi}(X, Y)$ for $1 \leq \xi<\omega_{1}$ (Theorem 3.3).

\section{Strictly SINGULAR OPERATORS}

We start by recalling some standard terminology and facts from descriptive set theory and Banach spaces. More information can be found in either [10] or [3]. For a non-empty set $X$ let $X^{<\mathbb{N}}$ denote the set containing all finite sequences of elements of $X$. If $X$ is a topological space then $X^{<\mathbb{N}}$ is a topological space as a direct sum of the spaces $X^{n}$ for all $n \in \mathbb{N}$. If $X$ is a set then a tree on $X$ is a subset $\mathcal{T}$ of $X^{<\mathbb{N}}$ such that if $\left(x_{1}, x_{2}, \ldots, x_{m}\right) \in \mathcal{T}$ (for some $m \in \mathbb{N}$ and $\left.x_{1}, \ldots, x_{m} \in X\right)$ then $\left(x_{1}, x_{2}, \ldots, x_{n}\right) \in \mathcal{T}$ for all $1 \leq n<m$. If $\mathcal{T}$ is a tree on a set $X$ then an infinite branch of $\mathcal{T}$ is an infinite sequence $\left(x_{n}\right)_{n \in \mathbb{N}}$ of elements of $\mathcal{T}$ such that $\left(x_{1}, \ldots x_{n}\right) \in \mathcal{T}$ for all $n \in \mathbb{N}$. A tree is called well founded if it does not contain infinite branches. If $\mathcal{T}$ is a tree on $X$ then the $\alpha$ derivative of $\mathcal{T}, \mathcal{T}^{(\alpha)}$, can be defined for any $\alpha<\omega_{1}$ after successively applying " $\alpha$ many trimmings of final nodes of $\mathcal{T}$ " (for the precise definition see either [3, pages 1010-1011] or [10, page 11]). The height of $\mathcal{T}, h(\mathcal{T})$, is the least ordinal $\alpha<\omega_{1}$ such that $\mathcal{T}^{(\alpha)}=\emptyset$, if such ordinals exist, else we set $h(\mathcal{T})=\omega_{1}$. The following is well known ([3, Theorem I.1.4]).

Theorem 2.1. Let $X$ be a Polish space, and $\mathcal{T}$ be an analytic well founded tree on $X$. Then $h(\mathcal{T})<\omega_{1}$.

For $1 \leq \xi<\omega_{1}$, the Schreier family, $S_{\xi}$ was introduced in [1], (see also [3, page 1038]), and contains certain finite subsets of $\mathbb{N}$. Let $\mathcal{L}(X, Y)$ denote the space of bounded linear operators from the Banach space $X$ to the Banach space $Y$. An operator $T \in \mathcal{L}(X, Y)$ is strictly singular, (denoted $T \in \mathcal{S S}(X, Y)$ ), if the restriction of $T$ to any infinite dimensional subspace of $X$ is not an isomorphism. In [2], the notion of $\mathcal{S}_{\xi}$ strictly singular operator is introduced (for $1 \leq \xi<\omega_{1}$ ) 
as follows. If $X, Y$ are Banach spaces, $T \in \mathcal{L}(X, Y)$ and $1 \leq \xi<\omega_{1}, T$ is $\mathcal{S}_{\xi}$ strictly singular (denoted by $T \in \mathcal{S S}_{\xi}(X, Y)$ ) if for every $\varepsilon>0$ and every basic sequence $\left(x_{n}\right)$ in $X$ there exists a set $F \in \mathcal{S}_{\xi}$ and $x \in \operatorname{Span}\left(x_{n}\right)_{n \in F}$ such that $\|T x\|<\varepsilon\|x\|$. Thus $\mathcal{S S}_{\xi}(X, Y)$ is a subset of $\mathcal{S S}(X, Y)$. Also, $([2]), \mathcal{S S}_{\xi}(X, Y) \subseteq \mathcal{S S}_{\zeta}(X, Y)$ if $1 \leq \xi<\zeta<\omega_{1}$. It is then proved in [2] that:

Theorem 2.2. Let $X, Y$ be separable Banach spaces. Then $\mathcal{S} \mathcal{S}(X, Y)=\bigcup_{\xi<\omega_{1}} \mathcal{S S}_{\xi}(X, Y)$.

Here we give the following refinement of Theorem 2.2.

Theorem 2.3. Let $Y$ and $Z$ be Banach spaces such that $Y$ is separable and let $Y^{*}$ denote the dual of $Y$. Then $\mathcal{S} \mathcal{S}\left(Y^{*}, Z\right)=\bigcup_{\xi<\omega_{1}} \mathcal{S S}_{\xi}\left(Y^{*}, Z\right)$.

Proof. Let $Y$ and $Z$ be as in the statement and assume that $T \in \bigcap_{\xi<\omega_{1}}\left(\mathcal{S S}_{\xi}\left(Y^{*}, Z\right)\right)^{c}$. Let $\mathcal{B}$ denote the set of normalized basic sequences in $Y^{*}, B a\left(Y^{*}\right)$ denote the unit ball of $Y^{*}$ and $S\left(Y^{*}\right)$ denote the unit sphere of $Y^{*}$ (i.e. the vectors of norm equal to 1 ). Let $\mathcal{Y}=$ $\left(B a\left(Y^{*}\right) \text {, weak }{ }^{*} \text { topology }\right)^{\mathbb{N}}$ and $\mathcal{S}=\left(S\left(Y^{*}\right) \text {, weak }{ }^{*} \text { topology }\right)^{\mathbb{N}}$. Then $\mathcal{Y}$ is a Polish space, $\left(S\left(Y^{*}\right)\right.$, weak ${ }^{*}$ topology $)$ is a Borel subset of $\left(B a\left(Y^{*}\right)\right.$, weak * topology) and thus $\mathcal{S}$ is a Borel subset of $\mathcal{Y}$.

We claim that $\mathcal{B}$ is a Borel subset of $\mathcal{S}$ and thus a Borel subset of $\mathcal{Y}$. Indeed, $\mathcal{B}=\bigcup_{k \in \mathbb{N}} \mathcal{B}_{k}$ where $\mathcal{B}_{k}$ denote the set of normalized basic sequences with basis constant at most $k$. To see that $\mathcal{B}_{k}$ is a Borel set, observe that for $\left(y_{n}^{*}\right) \in \mathcal{S}$,

$$
\left(y_{n}^{*}\right)_{n} \in \mathcal{B}_{k} \Leftrightarrow \forall N<M \text { in } \mathbb{N}, \forall\left(a_{n}\right) \in \mathbb{Q}^{<\mathbb{N}}, \forall r \in \mathbb{Q}^{+},\left\|\sum_{n=1}^{M} a_{n} y_{n}^{*}\right\|>r \text { or }\left\|\sum_{n=1}^{N} a_{n} y_{n}^{*}\right\| \leq k r .
$$

For fixed $N<M$ in $\mathbb{N},\left(a_{n}\right)_{n} \in \mathbb{Q}^{<\mathbb{N}}$ and $r \in \mathbb{Q}^{+}$, the sets $\left\{\left(y_{n}^{*}\right)_{n} \in \mathcal{Y}:\left\|\sum_{n=1}^{M} a_{n} y_{n}^{*}\right\|>r\right\}$ and $\left\{\left(y_{n}^{*}\right)_{n} \in \mathcal{Y}:\left\|\sum_{n=1}^{N} a_{n} y_{n}^{*}\right\| \leq k r\right\}$ are open and closed, respectively in $\mathcal{Y}$. Thus $\mathcal{B}_{k}$ is Borel and so $\mathcal{B}$ is a Borel subset of $\mathcal{S}$.

Let $\mathcal{K}$ be the Polish space $\mathbb{N} \times \mathbb{N} \times \mathcal{Y}$. Recall, that $\mathcal{K}^{<\mathbb{N}}$ denotes the direct sum of the spaces $\mathcal{K}^{n}$ (for $n \in \mathbb{N}$ ) and a tree on $\mathcal{K}$ will be a subset of $\mathcal{K}^{<\mathbb{N}}$. Define a tree $\mathcal{R}$ on $\mathcal{K}$ to be the following set:

$\left\{\left(m_{i}, \ell_{i},\left(y_{i, k}^{*}\right)_{k}\right)_{i=1}^{n} \in \mathcal{K}^{<\mathbb{N}}: m_{1}=\cdots=m_{n},\left(\ell_{1}<\cdots<\ell_{n}\right) \in \mathbb{N}^{<\mathbb{N}},\left(y_{1, k}^{*}\right)_{k}=\cdots=\left(y_{n, k}^{*}\right)_{k} \in \mathcal{B}\right\}$.

Note that a generic element of $\mathcal{R}$ will look like $\left(m, \ell_{i},\left(y_{k}^{*}\right)_{k}\right)_{i=1}^{n}$ where $m, n \in \mathbb{N},\left(\ell_{1}<\ell_{2}<\right.$ $\left.\cdots<\ell_{n}\right) \in \mathbb{N}^{n}$ and $\left(y_{k}^{*}\right)_{k} \in \mathcal{B}$. Obviously $\mathcal{R}$ is a closed subset of $(\mathbb{N} \times \mathbb{N} \times \mathcal{B})^{<\mathbb{N}}$ thus it is a Borel subset of $\mathcal{K}^{<\mathbb{N}}$, (since $(\mathbb{N} \times \mathbb{N} \times \mathcal{B})^{<\mathbb{N}}$ is a Borel subset of $\left.\mathcal{K}^{<\mathbb{N}}\right)$. For each $m \in \mathbb{N}$, define the following subtree of $\mathcal{R}$.

$$
\mathcal{T}=\left\{\left(m, \ell_{i},\left(y_{k}^{*}\right)_{k}\right)_{i=1}^{n} \in \mathcal{R}: \forall\left(a_{i}\right)_{i} \in \mathbb{Q}^{<\mathbb{N}},\left\|T \sum_{i=1}^{n} a_{i} y_{\ell_{i}}\right\|>\frac{1}{m}\left\|\sum_{i=1}^{n} a_{i} y_{\ell_{i}}\right\|\right\} .
$$


$\mathcal{T}$ is a Borel subtree of $\mathcal{R}$, (since $T$ is $\|\cdot\|$-weak* ${ }^{*}$ continuous and $\|\cdot\|$ is weak* lower semicontinuous). Since $T \in \bigcap_{\xi<\omega_{1}}\left(\mathcal{S S}_{\xi}\left(Y^{*}, Z\right)\right)^{c}$, for all $\xi<\omega_{1}$ there is an $m \in \mathbb{N}$ and $\left(y_{k}^{*}\right)_{k} \in \mathcal{B}$ such that for all $\left(\ell_{1}, \ldots, \ell_{n}\right) \in S_{\xi}$ we have that $\left(m, \ell_{i},\left(y_{k}^{*}\right)_{k}\right)_{i=1}^{n} \in \mathcal{T}$. The subtree $\mathcal{T}_{\left(y_{k}^{*}\right)_{k}, m}$ of $\mathcal{T}$ containing nodes of the form $\left(m, \ell_{i},\left(y_{k}^{*}\right)_{k}\right)_{i=1}^{n}$ with $\left(\ell_{1}, \ldots, \ell_{n}\right) \in S_{\xi}$ is order isomorphic to $S_{\xi}$. Thus for each $\xi<\omega_{1}$ the height of $\mathcal{T}$ is greater than or equal to $h\left(S_{\xi}\right)=\omega^{\xi}$. Whence $h(\mathcal{T})=\omega_{1}$. By Theorem 2.1, $\mathcal{T}$ is not well founded and thus there is an $m \in \mathbb{N},\left(y_{k}^{*}\right)_{k} \in \mathcal{B}$ and $\left(\ell_{i}\right)_{i=1}^{\infty}$ such that for all $n \in \mathbb{N}\left(m, \ell_{i},\left(y_{k}^{*}\right)_{k}\right)_{i=1}^{n} \in \mathcal{T}$. This implies that $\left.T\right|_{\left[y_{\ell_{i}}^{*}\right]_{i=1}^{\infty}}$ is an isomorphism and thus $T$ is not in $S S\left(Y^{*}, Z\right)$.

\section{StRictly COSINGUlAR OPERATORS}

For Banach spaces $X$ and $Y$ and an operator $T \in \mathcal{L}(X, Y)$, A. Pelczynski defined in [11] that $T$ is called strictly cosingular if for any subspace $Z$ of $Y$ of infinite codimension, the operator $Q_{Z} T$ is not onto, where $Q_{Z}$ is the canonical quotient map from $Y$ to $Y / Z$. We denote by $\mathcal{S C}(X, Y)$ the set of all strictly cosingular operators from $X$ to $Y$. Notice that by dualizing, $T \in \mathcal{L}(X, Y)$ is strictly cosingular if and only if for any infinite dimensional weak*closed subspace $W$ of $Y^{*}$, the restriction of $T^{*}$ on $W,\left.T^{*}\right|_{W}$, is not an isomorphism. Thus, in particular, if $T^{*} \in \mathcal{S S}\left(Y^{*}, X^{*}\right)$ then $T \in \mathcal{S C}(X, Y)$. The converse is in general false, as it can be seen from the inclusion operator from $c_{0}$ to $\ell_{\infty}$. Pelczynski proved in [11] that this operator is strictly cosingular but its adjoint is a projection from the dual of $\ell_{\infty}$ to $\ell_{1}$ which fails to be strictly singular. For Banach spaces $X$ and $Y$ let $S S_{*}(X, Y)$ denote the set of all operators $T \in \mathcal{L}(X, Y)$ such that $T^{*} \in \mathcal{S} \mathcal{S}\left(Y^{*}, X^{*}\right)$. For every $\xi<\omega_{1}$ define the classes $S S_{\xi, *}(X, Y)$ to contain all operators $T \in \mathcal{L}(X, Y)$ such that $T^{*} \in \mathcal{S S}_{\xi}\left(Y^{*}, X^{*}\right)$. Note that if $Y$ is separable then by Theorem $2.3, \mathcal{S S}_{*}(X, Y)=\bigcup_{\xi<\omega_{1}} \mathcal{S S}_{\xi, *}(X, Y)$. Thus, if the range space $Y$ is separable and $\mathcal{S C}(X, Y)=\mathcal{S S}_{*}(X, Y)$ then we obtain an answer to J. Diestel's question.

Recall that W.T. Gowers answered in the negative, in [8], the question of whether every Banach space contains a boundedly complete basic sequence or an isomorph $c_{0}$. Consequently V.P. Fonf characterized in [7] the class $\mathcal{K}$ of Banach spaces that contain either a boundedly complete basic sequence or $c_{0}$. Let $\mathcal{K}_{s}$ denote the class of Banach spaces such that all of their infinite dimensional closed subspaces contain a boundedly complete basic sequence or an isomorph of $c_{0}$. Many known Banach spaces belong to $\mathcal{K}_{s}$. For example, since every space with an unconditional basis contains $c_{0}, \ell_{1}$ or a reflexive subspace, we obtain that $\mathcal{K}_{s}$ contains all Banach spaces which are saturated with unconditional basic sequences. Also by the results of W.B. Johnson and H.P. Rosenthal, in [9, Theorem IV.1.(ii)], all separable dual spaces belong to $\mathcal{K}_{s}$. 
Theorem 3.1. Let $Y$ be separable and $Y^{*} \in \mathcal{K}_{s}$. Then for any Banach space $X, \mathcal{S C}(X, Y)=$ $\mathcal{S S}_{*}(X, Y)$. Thus by Theorem 2.3, $\mathcal{S C}(X, Y)=\bigcup_{\xi<\omega_{1}} \mathcal{S S}_{\xi, *}(X, Y)$.

Proof. Let $T \in \mathcal{S C}(X, Y)$ and suppose $T^{*}$ is not in $\mathcal{S S}\left(Y^{*}, X^{*}\right)$. Find a subspace $Z$ of $Y^{*}$ such $\left.T^{*}\right|_{Z}$ is an isomorphism. Our goal is to find an infinite dimensional weak* closed subspace preserved by $T^{*}$. This will contradict the fact that $T \in \mathcal{S C}(X, Y)$, and establish that $\mathcal{S C}(X, Y)=\mathcal{S S}_{*}(X, Y)$. The fact that $\mathcal{S C}(X, Y)=\bigcup_{\xi<\omega_{1}} \mathcal{S S}_{\xi, *}(X, Y)$ follows immediately from Theorem 2.3 since $Y$ is separable.

Since $Y^{*}$ is in $\mathcal{K}_{s}$, we first assume that $Z$ contains a normalized boundedly complete basic sequence, call it $\left(y_{n}^{*}\right)$. Since $Y$ is separable, the weak* topology on the unit ball of $Y^{*}$ is metrizable, thus $\left(y_{n}^{*}\right)$ has a weak* convergent subsequence. Let $\left(z_{n}^{*}\right)$ be the difference sequence of that weak* convergent subsequence of $\left(y_{n}^{*}\right)$. Then $\left(z_{n}^{*}\right)$ is weak* null seminormalized boundedly complete basic sequence in $Z$. By [9, Theorem III.1], since $Y$ is separable, $\left(z_{n}^{*}\right)$ has a further subsequence $\left(z_{k_{n}}^{*}\right)$ which is weak* basic (and boundedly complete). By [9, Proposition II.1], the weak ${ }^{*}$ and norm closure of the linear span of $\left(z_{k_{n}}^{*}\right)$ coincide and thus $Z$ contains an infinite dimensional weak ${ }^{*}$ closed subspace. The restriction of $T^{*}$ on that subspace is an isomorphism.

In the second case we assume that $Z$ contains and isomorph of $c_{0}$. We recall that H.P. Rosenthal proved in [12, Theorem 1.3] that if an operator originates from a Banach space which is complemented in its second dual and preserves an isomorphic copy of $c_{0}$ then it preserves an isomorphic copy of $\ell_{\infty}$. Since the dual Banach space $Y^{*}$ is complemented in its second dual, by the above result of Rosenthal, there exists a subspace $W$ of $Y^{*}$ which is isomorphic to $\ell_{\infty}$ such that the restriction of $T^{*}$ on $W$ is an isomorphic embedding. Obviously $W$ contains a weak* closed subspace of $Y^{*}$ (consider, for example, a reflexive subspace of $W$ ). The restriction of $T^{*}$ on that weak* closed subspace is also an isomorphism.

The assumption on $Y^{*}$ in Theorem 3.1 can be eliminated if we work with the class of $\left(\mathcal{A}_{i}\right)_{i \in I}$ strictly cosingular operators instead of the class of strictly cosingular operators. We now motivate the definition of $\left(\mathcal{A}_{i}\right)_{i \in I}$ strictly cosingular operators by examining the definition of strictly cosingular operators. Let $X, Y$ be Banach spaces and $T \in \mathcal{L}(X, Y)$. Since every weak* closed subspace of $Y^{*}$ contains the weak* closed linear span of some basic sequence we have that $T \in \mathcal{S C}(X, Y)$ if and only if for any basic sequence $\left(y_{n}^{*}\right)_{n}$ in $Y^{*},\left.T^{*}\right|_{\widehat{\operatorname{Span}}\left(y_{n}^{*}\right)}$ is not an isomorphism (where $\operatorname{Span}\left(y_{n}^{*}\right)$ denotes the linear span of $\left(y_{n}^{*}\right)$ and $\widetilde{\operatorname{Span}}\left(y_{n}^{*}\right)$ denotes the weak* closure of the linear span of $\left(y_{n}^{*}\right)$ ). When attempting to define subclasses of strictly cosingular operators in a way similar to the definition of $S_{\xi}$ strictly singular operators, we encounter an immediate problem. Namely, whether or not $\left.T^{*}\right|_{\operatorname{Span}\left(y_{n}^{*}\right)}$ is an isomorphism is not characterized by whether or not $\left.T^{*}\right|_{\operatorname{Span}\left(y_{n}^{*}\right)_{n \in F}}$ is an isomorphism with the same isomorphism constant for 
any finite subset $F$ of $\mathbb{N}$. A natural way to compensate for this is to consider complements of the finite sets. Of course, $\left.T^{*}\right|_{\widetilde{\operatorname{Span}}\left(y_{n}^{*}\right)}$ is not an isomorphism for all basic sequences $\left(y_{n}^{*}\right)$ in $Y^{*}$, if and only if for all basic sequence $\left(y_{n}^{*}\right)$ in $Y^{*}$ and for every finite subset $I$ of $\mathbb{N},\left.T^{*}\right|_{\operatorname{Span}\left(y_{n}^{*}\right)_{n \notin I}}$ is not an isomorphism. A new problem arises: If for every basic sequence $\left(y_{n}^{*}\right)$ in $Y^{*}$ one examines the restriction of $T^{*}$ on $\widetilde{\operatorname{Span}}\left(y_{n}^{*}\right)_{n \notin I}$ where $I$ belong to a fixed class $\mathcal{I}$ of finite subset of $\mathbb{N}$ (e.g. $\mathcal{I}=S_{\xi}$ for some $\left.\xi<\omega_{1}\right)$, then the fact that we examine every basic sequence $\left(y_{n}^{*}\right)_{n}$, makes the class $\mathcal{I}$ unimportant, since $\left(y_{n}^{*}\right)_{n \notin I}$ is another basic sequence in $Y^{*}$. We fix this problem by fixing a family $\left(\mathcal{A}_{i}\right)_{i \in I}$ of sets $\mathcal{A}_{i}$ of basic sequences in $Y^{*}$ and examining whether, for every $i \in I$ there exists a basic sequence $\left(y_{n}^{*}\right) \in \mathcal{A}_{i}$ such that for all finite subsets $F$ of positive integers,

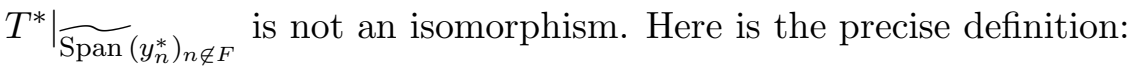

Definition 3.2. Let $X, Y$ be Banach spaces and $T \in \mathcal{L}(X, Y)$. Let $I$ be an index set and for every $i \in I$ let $\mathcal{A}_{i}$ be a set of basic sequences in the dual space $Y^{*}$ of $Y$. Fix $1 \leq \xi<\omega_{1}$.

(1) We say that $T$ is $\left(\mathcal{A}_{i}\right)_{i \in I}$ strictly cosingular, (denoted by $T \in\left(\mathcal{A}_{i}\right)_{i \in I}-\mathcal{S C}(X, Y)$ ), if and only if for every $i \in I$ there exists $\left(y_{n}^{*}\right)_{n} \in \mathcal{A}_{i}$ and an infinite subset $N$ of the positive integers such that for every finite subset $F$ of $N$ we have that $\left.T^{*}\right|_{\widehat{\operatorname{Span}}\left(y_{n}^{*}\right)_{n \notin F}}$ is not an isomorphism.

(2) We say that $T$ is $\left(\mathcal{A}_{i}\right)_{i \in I^{-}} S_{\xi}$ strictly cosingular , (denoted by $\left.T \in\left(\mathcal{A}_{i}\right)_{i \in I}-\mathcal{S C}_{\xi}(X, Y)\right)$, if and only if for every $i \in I$ there exists $\left(y_{n}^{*}\right)_{n} \in \mathcal{A}_{i}$ and an infinite subset $N$ of the positive integers such that for every finite subset $F$ of $N$ with $F \in S_{\xi}$ we have that $\left.T^{*}\right|_{\operatorname{Span}\left(y_{n}^{*}\right)_{n \notin F}}$ is not an isomorphism.

First note that for Banach spaces $X$ and $Y$ and any families $\left(\mathcal{A}_{i}\right)_{i \in I}$ of basic sequences of $Y^{*}$, we have $\mathcal{S C}(X, Y) \subseteq\left(\mathcal{A}_{i}\right)_{i \in I^{-}} \mathcal{S C}(X, Y)$. Also note that for a specific choice of $\left(\mathcal{A}_{i}\right)_{i \in I}$, we have that $\mathcal{S C}(X, Y)=\left(\mathcal{A}_{i}\right)_{i \in I^{-}} \mathcal{S C}(X, Y)$. Indeed, consider the set $\mathcal{W}$ of all weak* closed infinite dimensional subspace of $Y^{*}$, and for every $W \in \mathcal{W}$, consider the set $\mathcal{A}_{W}$ of all normalized basic sequences in $W$. Then it is easy to see that $\left(\mathcal{A}_{W}\right)_{W \in \mathcal{W}} \mathcal{S C}(X, Y)=\mathcal{S C}(X, Y)$. Also, $\left(\mathcal{A}_{W}\right)_{W \in \mathcal{W}^{-}} \mathcal{S C}_{\xi}(X, Y)=\mathcal{S C}(X, Y)$ for all $\xi<\omega_{1}$ thus the next Theorem 3.3 does not give new results for strictly cosingular operators.

It is obvious that for Banach spaces $X, Y$, a family $\left(\mathcal{A}_{i}\right)_{i \in I}$ of sets of basic sequences in $Y^{*}$ and an ordinal $1 \leq \xi<\omega_{1}$ we have that

$$
\left(\mathcal{A}_{i}\right)_{i \in I^{-}} \mathcal{S C}(X, Y) \subseteq\left(\mathcal{A}_{i}\right)_{i \in I^{-}} \mathcal{S} \mathcal{C}_{\xi}(X, Y)
$$

Also notice that for ordinals $1 \leq \xi<\zeta<\omega_{1}$ we have that

$$
\left(\mathcal{A}_{i}\right)_{i \in I^{-}} \mathcal{S C}_{\zeta}(X, Y) \subseteq\left(\mathcal{A}_{i}\right)_{i \in I^{-}} \mathcal{S C}_{\xi}(X, Y)
$$


For the last inclusion, let $T \in\left(\mathcal{A}_{i}\right)_{i \in I^{-}} \mathcal{S C}_{\zeta}(X, Y)$ and for $i \in I$ let a basic sequence $\left(y_{n}^{*}\right)_{n} \in \mathcal{A}_{i}$ and an infinite subset $N$ of positive integers such that for all $F \subset N$ with $F \in S_{\zeta},\left.T^{*}\right|_{\widehat{\operatorname{Span}}\left(y_{n}^{*}\right)_{n \notin F}}$ is not an isomorphism. There exists an infinite subset $M$ of $N$ such that for every $F \subset M$ with $F \in S_{\xi}$ we have that $F \in S_{\zeta}([3])$. Thus for every $F \subset M$ with $F \in S_{\xi},\left.T^{*}\right|_{\operatorname{Span}\left(y_{n}^{*}\right)_{n \notin F}}$ is not an isomorphism. Thus $T \in\left(\mathcal{A}_{i}\right)_{i \in I^{-}} \mathcal{S C}_{\xi}(X, Y)$.

The following result is the analogue of Theorems 2.2 and 2.3 for $\left(\mathcal{A}_{i}\right)_{i \in I}$ strictly cosingular operators.

Theorem 3.3. Let $X$ and $Y$ be Banach spaces such that $Y$ is separable, and $\left(\mathcal{A}_{i}\right)_{i \in I}$ be a family an analytic sets of basic sequences in $Y^{*}$. Then $\left(\mathcal{A}_{i}\right)_{i \in I}-\mathcal{S C}(X, Y)=\bigcap_{\xi<\omega_{1}}\left(\mathcal{A}_{i}\right)_{i \in I^{-}} \mathcal{S C}_{\xi}(X, Y)$.

Proof. It suffices to show that $\bigcap_{\xi<\omega_{1}}\left(\mathcal{A}_{i}\right)_{i \in I^{-}} \mathcal{S} \mathcal{C}_{\xi}(X, Y) \subset\left(\mathcal{A}_{i}\right)_{i \in I^{-}} \mathcal{S C}(X, Y)$. Fix an operator $T \in \bigcap_{\xi<\omega_{1}}\left(\mathcal{A}_{i}\right)_{i \in I^{-}} \mathcal{S C}_{\xi}(X, Y)$ and $i_{0} \in I$. As in the proof of Theorem 2.3, let $\mathcal{B}$ denote the set of normalized basic sequences in $Y^{*}, B a\left(Y^{*}\right)$ denote the unit ball of $Y^{*}, \mathcal{Y}$ denote the Polish space $B a\left(Y^{*}\right)^{\mathbb{N}}$ with the product of the weak* topology. Let $\mathcal{K}$ be the Polish space $\mathbb{N} \times \mathcal{Y}$. Recall, that $\mathcal{K}^{<\mathbb{N}}$ denotes the direct sum of the spaces $\mathcal{K}^{n}$ (for $n \in \mathbb{N}$ ) and a tree on $\mathcal{K}$ will be a subset of $\mathcal{K}^{<\mathbb{N}}$. Define a tree $\mathcal{R}$ on $\mathcal{K}$ as follows:

$$
\mathcal{R}=\left\{\left(\ell_{i},\left(y_{i, k}^{*}\right)_{k}\right)_{i=1}^{n} \in \mathcal{K}^{<\mathbb{N}}:\left(\ell_{1}<\cdots<\ell_{n}\right) \in \mathbb{N}^{<\mathbb{N}},\left(y_{1, k}^{*}\right)_{k}=\cdots=\left(y_{n, k}^{*}\right)_{k} \in \mathcal{B}\right\} .
$$

Note that a generic element of $\mathcal{R}$ will look like $\left(\ell_{i},\left(y_{k}^{*}\right)_{k}\right)_{i=1}^{n}$ where $n \in \mathbb{N},\left(\ell_{1}<\ell_{2}<\cdots<\right.$ $\left.\ell_{n}\right) \in \mathbb{N}^{n}$ and $\left(y_{k}^{*}\right)_{k} \in \mathcal{B}$. It has been shown in the proof of Theorem 2.3 that $\mathcal{B}$ is a Borel subset of $\mathcal{Y}$. Thus $(\mathbb{N} \times \mathcal{B})^{<\mathbb{N}}$ is a Borel subset of $\mathcal{K}^{<\mathbb{N}}=(\mathbb{N} \times \mathcal{Y})^{<\mathbb{N}}$. Obviously $\mathcal{R}$ is a closed subset of $(\mathbb{N} \times \mathcal{B})^{<\mathbb{N}}$ thus it is a Borel subset of $\mathcal{K}^{<\mathbb{N}}$. Define the following two subtrees of $\mathcal{R}$.

$$
\begin{gathered}
\mathcal{T}=\left\{\left(\ell_{i},\left(y_{k}^{*}\right)_{k}\right)_{i=1}^{n} \in \mathcal{R}:\left.T^{*}\right|_{\widehat{\operatorname{Span}}\left(y_{k}^{*}\right)_{k \notin\left\{\ell_{1}, \ldots, \ell_{n}\right\}}} \text { is not an isomorphism }\right\} \\
\mathcal{T}_{i_{0}}=\left\{\left(\ell_{i},\left(y_{k}^{*}\right)_{k}\right)_{i=1}^{n} \in \mathcal{R}:\left(y_{k}^{*}\right)_{k} \in \mathcal{A}_{i_{0}}\right\}
\end{gathered}
$$

Both $\mathcal{T}$ and $\mathcal{T}_{i_{0}}$ are trees on $\mathcal{K}$. The main component of the proof will be to show that $\tilde{\mathcal{T}}=\mathcal{T} \cap \mathcal{T}_{i_{0}}$ is an analytic subtree of $\mathcal{R}$, and thus an analytic tree on $\mathcal{K}$.

In order to see that $\mathcal{T}_{i_{0}}$ is an analytic tree, define the map $\pi: \mathcal{R} \rightarrow \mathcal{B}$ by $\pi\left(\left(\ell_{i},\left(y_{k}^{*}\right)_{k}\right)_{i=1}^{n}\right)=$ $\left(y_{k}^{*}\right)_{k}$ where $\left(\left(\ell_{i},\left(y_{k}^{*}\right)_{k}\right)_{i=1}^{n}\right)$ is a generic element of $\mathcal{R}$. Obviously this map is continuous, and $\mathcal{T}_{i_{0}}=\pi^{-1}\left(\mathcal{A}_{i_{0}}\right)$. Since $\mathcal{A}_{i_{0}}$ is an analytic set, we obtain that $\mathcal{T}_{i_{0}}$ is analytic (see e.g. [10, Proposition 14.3(ii)]).

To demonstrate that $\mathcal{T}$ is analytic, we wish to write it as a countable intersection of analytic sets. Let,

$$
\begin{aligned}
\mathcal{C}_{\varepsilon}=\left\{\left(\left(\ell_{i},\left(y_{k}^{*}\right)_{k}\right)_{i=1}^{n}, y^{*}\right) \in\right. & \mathcal{R} \times\left(B a\left(Y^{*}\right), \text { weak }^{*} \text { topology }\right): y^{*} \in \widetilde{\operatorname{Span}}\left(y_{k}^{*}\right)_{k \notin\left\{\ell_{1}, \ldots, \ell_{n}\right\}} \\
& \text { and } \left.\left\|T^{*} y^{*}\right\|<\varepsilon\left\|y^{*}\right\|\right\} .
\end{aligned}
$$


In this definition, as well as the rest of the proof, $B a\left(Y^{*}\right)$ will be equipped with the weak* topology. Clearly, $\mathcal{T}=\bigcap_{\varepsilon \in \mathbb{Q}^{+}} \operatorname{proj}_{\mathcal{R}} \mathcal{C}_{\varepsilon}$ where $\operatorname{proj}_{\mathcal{R}}$ denotes the projection to $\mathcal{R}$. Since $\mathcal{R}$ is a Borel subset of $\mathcal{K}^{<\mathbb{N}}$ there exists a finer Polish topology on $\mathcal{K}^{<\mathbb{N}}$ making $\mathcal{R}$ a clopen set and having the same Borel sets as the ordinary topology of $\mathcal{K}^{<\mathbb{N}}$ ([10, Theorem 13.1]). Considering this finer topology on $\mathcal{R}$ we have that $\mathcal{R} \times\left(B a(Y)\right.$, weak ${ }^{*}$ topology) is Polish. Thus once we show that $\mathcal{C}_{\varepsilon}$ is Borel we obtain that $\operatorname{proj}_{\mathcal{R}} \mathcal{C}_{\varepsilon}$ is analytic $([10$, Exercise 14.3]) hence $\mathcal{T}$ is analytic. Decompose $\mathcal{C}_{\varepsilon}$ as the intersection of the following two sets,

$$
\begin{gathered}
\mathcal{C}_{\varepsilon}^{1}=\left\{\left(\left(\ell_{i},\left(y_{k}^{*}\right)_{k}\right)_{i=1}^{n}, y^{*}\right) \in \mathcal{R} \times B a\left(Y^{*}\right),:\left\|T^{*} y^{*}\right\|<\varepsilon\left\|y^{*}\right\|\right\}, \\
\mathcal{C}^{2}=\left\{\left(\left(\ell_{i},\left(y_{k}^{*}\right)_{k}\right)_{i=1}^{n}, y^{*}\right) \in \mathcal{R} \times B a\left(Y^{*}\right): y^{*} \in \widetilde{\operatorname{Span}}\left(y_{k}^{*}\right)_{k \notin\left\{\ell_{1}, \ldots, \ell_{n}\right\}}\right\} .
\end{gathered}
$$

For $\left(\left(\ell_{i},\left(y_{k}^{*}\right)_{k}\right)_{i=1}^{n}, y^{*}\right) \in \mathcal{R} \times B a\left(Y^{*}\right)$ notice that

$$
\left(\left(\ell_{i},\left(y_{k}^{*}\right)_{k}\right)_{i=1}^{n}, y^{*}\right) \in \mathcal{C}_{\varepsilon}^{1} \Leftrightarrow \forall r \in \mathbb{Q}^{+},\left\|y^{*}\right\|>r \text { or }\left\|T^{*} y^{*}\right\| \leq \varepsilon r .
$$

Hence, $\mathcal{C}_{\varepsilon}^{1}$ is a Borel subset of $\mathcal{R} \times B a\left(Y^{*}\right)$ (by the weak* lower semicontinuity of $\|\cdot\|$ ).

Now we demonstrate that $\mathcal{C}^{2}$ is a Borel set. Let $F\left(Y^{*}\right)$ denote the topological space of the weak* closed subsets of $Y^{*}$. This is called "the Effros-Borel space of the topological space $\left(Y^{*}\right.$, weak ${ }^{*}$ topology)", (see [10, section 12.C]), and its basic open sets have the form $V_{U}:=$ $\left\{F \in F\left(Y^{*}\right): F \cap U \neq \emptyset\right\}$ (where $U$ is an arbitrary weak* open subset of $Y^{*}$ ). Define the map, $\psi: \mathcal{R} \times B a\left(Y^{*}\right) \rightarrow F\left(Y^{*}\right)$, by

$$
\psi\left(\left(i,\left(y_{n}^{*}\right)_{n}\right)_{i \in I}, y^{*}\right)=\left\{z^{*}-y^{*}: z^{*} \in \widetilde{\operatorname{Span}}\left(y_{n}^{*}\right)_{n \notin I}\right\} .
$$

We claim that $\psi$ is continuous. Indeed, fix a weak* open subset $U$ in $Y^{*}$ and notice that,

$$
\begin{aligned}
\psi^{-1}\left(V_{U}\right) & =\left\{\left(\left(i,\left(y_{k}^{*}\right)_{k}\right)_{i \in I}, y^{*}\right) \in \mathcal{R} \times B a\left(Y^{*}\right):\left\{z^{*}-y^{*}: z^{*} \in \widetilde{\operatorname{Span}}\left(y_{n}^{*}\right)_{n \notin I}\right\} \cap U \neq \emptyset\right\} \\
& =\left\{\left(\left(i,\left(y_{k}^{*}\right)_{k}\right)_{i \in I}, y^{*}\right) \in \mathcal{R} \times B a\left(Y^{*}\right):\left\{z^{*}-y^{*}: z^{*} \in \operatorname{Span}\left(y_{n}^{*}\right)_{n \notin I}\right\} \cap U \neq \emptyset\right\},
\end{aligned}
$$

(since $U$ is weak* open, $\left.\operatorname{Span}\left(y_{n}^{*}\right)_{n \notin I} \cap\left(y^{*}+U\right) \neq \emptyset \Leftrightarrow \widetilde{\operatorname{Span}}\left(y_{n}^{*}\right)_{n \notin I} \cap\left(y^{*}+U\right) \neq \emptyset\right)$. Thus

$$
\psi^{-1}\left(V_{U}\right)=\bigcup_{I \in \mathbb{N}<\mathbb{N},\left(a_{i}\right) \in \mathbb{R}<\mathbb{N}} \mathcal{C}_{I,\left(a_{i}\right)}
$$

where for fixed $I \in \mathbb{N}^{<\mathbb{N}}$ and $\left(a_{i}\right) \in \mathbb{R}^{<\mathbb{N}}$,

$$
\mathcal{C}_{I,\left(a_{i}\right)}:=\left\{\left(\left(i,\left(y_{k}^{*}\right)_{k}\right)_{i \in I}, y^{*}\right) \in \mathcal{R} \times B a\left(Y^{*}\right): \sum_{i \notin I} a_{i} y_{i}^{*}-y^{*} \in U\right\} .
$$

Since $U$ is a weak* open subset of $Y^{*}, \mathcal{C}_{I,\left(a_{i}\right)}$ is an open subset of $\mathcal{R} \times B a\left(Y^{*}\right)$, showing that $\psi$ is continuous. Since $Y$ is separable, $\{0\}$ is a weak* closed set thus the set $\left\{F \in F\left(Y^{*}\right): 0 \in F\right\}$ is $G_{\delta}$ in $F\left(Y^{*}\right)$ and therefore

$$
\mathcal{C}^{2}=\psi^{-1}\left(\left\{F \in F\left(Y^{*}\right): 0 \in F\right\}\right)=\left\{\left(\left(i,\left(y_{k}^{*}\right)_{k}\right)_{i \in I}, y^{*}\right) \in \mathcal{R} \times B^{w^{*}}: y^{*} \in \widetilde{\operatorname{Span}}\left(y_{n}^{*}\right)_{n \notin I}\right\}
$$

is Borel. 
Having determined that $\mathcal{C}^{2}$ and $\mathcal{C}_{\varepsilon}^{1}$ are both Borel, it follows that $\mathcal{C}_{\varepsilon}$ is Borel for each $\varepsilon \in \mathbb{Q}^{+}$. Finally $\widetilde{\mathcal{T}}$ is an analytic tree on $\mathcal{K}$.

By our assumption, for each $\xi<\omega_{1}$ there exist a $\left(y_{n}^{*}\right) \in \mathcal{A}_{i_{0}}$ and an infinite subset $N$ of $\mathbb{N}$ such that for any $\left(\ell_{1}<\ldots<\ell_{n}\right) \subset N$ with $\left(\ell_{1}, \ldots, \ell_{n}\right) \in S_{\xi},\left(\ell_{i},\left(y_{k}^{*}\right)_{k}\right)_{i=1}^{n} \in \tilde{\mathcal{T}}$. The subtree $\widetilde{\mathcal{T}}_{\left(y_{n}^{*}\right)_{n}, N}$ of $\widetilde{\mathcal{T}}$ containing all the nodes of the form $\left(\ell_{i},\left(y_{k}^{*}\right)_{k}\right)_{i=1}^{n}$ with $\left(\ell_{1}<\ldots<\ell_{n}\right) \subset N$ and $\left(\ell_{1}, \ldots, \ell_{n}\right) \in S_{\xi}$ is order isomorphic to $S_{\xi}$. Thus, for each $\xi$ the height of the tree $\widetilde{\mathcal{T}}$ is greater that or equal to $h\left(S_{\xi}\right)=\omega^{\xi}$. Whence, $h(\widetilde{\mathcal{T}})=\omega_{1}$. By Theorem 2.1, conclude that $\widetilde{\mathcal{T}}$ is not well founded. Let $\left(y_{n}^{*}\right)_{n} \in \mathcal{A}_{i_{0}}$ and $\left(\ell_{i}\right)_{i=1}^{\infty}$ be an increasing sequence of positive integers such that for all $m \in \mathbb{N},\left(\ell_{i},\left(y_{k}^{*}\right)_{k}\right)_{i=1}^{m} \in \widetilde{\mathcal{T}}$. Therefore, if $F$ is any finite subset of $\left(\ell_{i}\right)_{i=1}^{\infty} \subset \mathbb{N},\left.T^{*}\right|_{\operatorname{Span}\left(y_{n}^{*}\right)_{n \notin F}}$ is not an isomorphism. Since the above is true for all $i_{0} \in I, T \in\left(\mathcal{A}_{i}\right)_{i \in I^{-}} \mathcal{S C}(X, Y)$.

We now give some examples of analytic sets of basic sequences in a dual Banach space $Y^{*}$ for separable $Y$. Such sets can be used for $\mathcal{A}_{i}$ in defining $\left(\mathcal{A}_{i}\right)_{i \in I^{-}} \mathcal{S C}(X, Y)$. Bossard, in [4], shows that for a separable Banach space $Z$, the set of all boundedly complete and the set of all shrinking basic sequence is coanalytic (the complement of an analytic set) non-Borel as a subset of $Z^{\mathbb{N}}$.

Remark 3.4. Assume that $Y$ is a separable Banach space. Let $W$ be a fixed infinite dimensional weak ${ }^{*}$ closed subspace of $Y^{*},\left(z_{n}^{*}\right)$ be a fixed basic sequence in $Y^{*}$ and $\mathcal{A}$ be a fixed analytic set of basic sequences in $Y^{*}$. Let $\mathcal{Y}$ be the Polish space $\left(B a\left(Y^{*}\right) \text {, weak } k^{*} \text { topology }\right)^{\mathbb{N}}$.

(1) The set $\mathcal{B}_{W}$, of normalized basic sequences in $W$ is a Borel subset of $\mathcal{Y}$.

(2) If $W$ has infinite codimension in $Y^{*}$ then the set $\mathcal{B}_{W^{c}}$ of all normalized basic sequences $\left(y_{n}^{*}\right)$ in $Y^{*}$ with $y_{n}^{*} \notin W$ for all $n \in \mathbb{N}$, is a Borel subset of $\mathcal{Y}$.

(3) The set $\mathcal{B}_{u}$, of all unconditional basic sequences is a Borel subset of $\mathcal{Y}$.

(4) The set $\mathcal{A}_{\left(z_{n}^{*}\right)}^{\text {sub }}$ of all subsequences of $\left(z_{n}^{*}\right)$ is an analytic subset of $\mathcal{Y}$.

(5) The set $\mathcal{A}_{\left(z_{n}^{*}\right)}^{\text {block }}$ of all block sequences of $\left(z_{n}^{*}\right)$ is an analytic subset of $\mathcal{Y}$.

(6) The set $\mathcal{A}_{n b c}$, of all non-boundedly complete basic sequences in $Y^{*}$ is an analytic subset of $\mathcal{Y}$.

(7) The set $\mathcal{A}_{b o}$, of all sequences in $\mathcal{A}$ with biorthogonal vectors in $Y$ is an analytic subset of $\mathcal{Y}$.

Proof. (1) is proved in Theorem 2.3. (2) is obvious since $Y^{*} \backslash W$ is a weak ${ }^{*}$ open set.

(3) This follows from writing $\mathcal{B}_{u}$ as,

$$
\begin{aligned}
\mathcal{B}_{u} & =\left\{\left(y_{n}^{*}\right)_{n} \in \mathcal{B}: \exists k \in \mathbb{N}, \forall\left(a_{i}\right)_{i} \in \mathbb{Q}^{<\mathbb{N}} \forall F \text { finite subset of } \mathbb{N},\left\|\sum_{i \in F} a_{i} y_{i}^{*}\right\|<k\left\|\sum_{i} a_{i} y_{i}^{*}\right\|\right\} \\
& =\bigcup_{k \in \mathbb{N}} \bigcap_{\left(a_{i}\right)_{i} \in \mathbb{Q}^{<\mathbb{N}}} \bigcap_{\substack{F \subset \mathbb{N} \\
\text { finite }}}\left\{\left(y_{n}^{*}\right)_{n} \in \mathcal{B}: \forall r \in \mathbb{Q}^{+},\left\|\sum_{i} a_{i} y_{i}^{*}\right\|>r \text { or }\left\|\sum_{i \in F} a_{i} y_{i}^{*}\right\| \leq k r\right\} .
\end{aligned}
$$


(4) We have that $\left(w_{n}^{*}\right) \in \mathcal{A}_{\left(z_{n}^{*}\right)}^{\text {sub }}$ if and only if there exists $k_{1}<k_{2}<\cdots$ an increasing sequence of positive integers such that $w_{n}^{*}=z_{k_{n}}^{*}$ for all $n \in \mathbb{N}$. The set $[\mathbb{N}]^{\mathbb{N}}$ of the increasing sequences of integers endowed with the coordinate-wise convergence is a Polish space, thus the result follows from [10, Exercise 14.3 (ii)].

(5) We have that $\left(w_{n}^{*}\right) \in \mathcal{B}_{\left(z_{n}^{*}\right)}^{\text {block }}$ if and only if there exists $k_{1}<k_{2}<\cdots$ an increasing sequence of integers and $\left(a_{i}\right) \in \mathbb{R}^{\mathbb{N}}$ such that $w_{n}^{*}=\sum_{i=k_{n}}^{k_{n+1}-1} a_{i} z_{i}^{*}$. Since $[\mathbb{N}]^{\mathbb{N}} \times \mathbb{R}^{\mathbb{N}}$ endowed with the coordinate-wise convergence is a Polish space, the result follows from [10, Exercise 14.3 (ii)].

(6) Set $\mathcal{B}=\mathcal{B}_{Y^{*}}($ see part (1)) and

$$
\mathcal{C}_{n b c}:=\left\{\left(\left(y_{n}^{*}\right)_{n},\left(a_{n}\right)_{n}\right) \in \mathcal{B} \times \mathbb{R}^{\mathbb{N}}: \exists M, \sup _{N}\left\|\sum_{n=1}^{N} a_{n} y_{n}^{*}\right\| \leq M \&\left(\sum_{n=1}^{N} a_{n} y_{n}^{*}\right)_{N} \text { is not Cauchy }\right\} .
$$

Observe that $\mathcal{A}_{n b c}=\operatorname{proj}_{\mathcal{B}} \mathcal{C}_{n b c}$ (the projection on $\mathcal{B}$ ). Therefore, it is enough to show that $\mathcal{C}_{n b c}$ is a Borel subset of $\mathcal{B} \times \mathbb{R}^{\mathbb{N}}$ (since we can assume that $\mathcal{B}$ is a Polish space by $[10$, Theorem 13.1]). For each $M \in \mathbb{N}$, the set

$$
\mathcal{C}^{M}=\left\{\left(\left(y_{n}^{*}\right)_{n},\left(a_{n}\right)_{n}\right) \in \mathcal{B} \times \mathbb{R}^{\mathbb{N}}: \sup _{N}\left\|\sum_{i=n}^{N} a_{n} y_{n}^{*}\right\| \leq M\right\}
$$

is a closed in $\mathcal{B} \times \mathbb{R}^{\mathbb{N}}$. Thus $\bigcup_{M \in \mathbb{N}} \mathcal{C}^{M}$ is Borel. Additionally,

$$
\begin{aligned}
\mathcal{D}=\left\{\left(\left(y_{i}^{*}\right)_{i},\left(a_{i}\right)_{i}\right) \in \mathcal{B} \times \mathbb{R}^{\mathbb{N}}:\left(\sum_{i=1}^{N} a_{i} y_{i}^{*}\right)_{N} \text { is not Cauchy }\right\} & \\
= & \left\{\left(\left(y_{i}^{*}\right)_{i},\left(a_{i}\right)_{i}\right) \in \mathcal{B} \times \mathbb{R}^{\mathbb{N}}: \exists r \in \mathbb{Q}^{+}, \forall n \in \mathbb{N}, \exists n<m \in \mathbb{N},\left\|\sum_{i=n}^{m} a_{i} y_{i}^{*}\right\|>r\right\} \\
& =\bigcup_{r \in \mathbb{Q}^{+}} \bigcap_{n \in \mathbb{N}} \bigcup_{n<m \in \mathbb{N}}\left\{\left(\left(y_{i}^{*}\right)_{i},\left(a_{i}\right)_{i}\right) \in \mathcal{B} \times \mathbb{R}^{\mathbb{N}}:\left\|\sum_{i=n}^{m} a_{i} y_{i}^{*}\right\|>r\right\}
\end{aligned}
$$

is Borel. Thus, $\mathcal{C}_{n b c}=\mathcal{D} \bigcap\left(\bigcup_{M \in \mathbb{N}} \mathcal{C}^{M}\right)$ is Borel.

(7) Set $\mathcal{C}_{b o}:=\left\{\left(\left(y_{n}^{*}\right)_{n},\left(y_{n}\right)_{n}\right) \in \mathcal{Y} \times Y^{\mathbb{N}}: y_{k}^{*}\left(y_{n}\right)=\delta_{k, n}\right\}$. Then $\mathcal{C}_{b o}$ is a closed, and hence trivially a Borel subset of $\mathcal{Y} \times Y^{\mathbb{N}}$. $\mathcal{A}_{b o}$ is analytic since $\mathcal{A}_{b o}=\operatorname{proj}_{\mathcal{Y}} \mathcal{C}_{b o}$ (the projection on $\mathcal{Y}$ ).

Finally we present an example in order to illustrate Theorem 3.3. Let $D$ be the dyadic tree $\{\emptyset\} \cup\{0,1\}^{<\mathbb{N}}$ and consider the space $X=\ell_{1}(D)$. Let $\left\{e_{\alpha}: \alpha \in D\right\}$ denote the basis of $\mathrm{X}$ which is ordered as: $e_{\emptyset}, e_{0}, e_{1}, e_{0,0}, e_{0,1}, e_{1,0}, e_{1,1}, e_{0,0,1}$, etc. Fix $\omega_{1}$ many infinite branches of $D$, and enumerate them as $\left\{b_{\xi}: \xi<\omega_{1}\right\}$. For fixed $\xi<\omega_{1}$, let $\left(e_{\xi, n}\right)_{n}$ be the increasing enumeration of the set $\left\{e_{\alpha}: \alpha \in b_{\xi}\right\}$. Also for every $\xi<\omega_{1}$ let $F_{\xi}$ be a fixed finite set which does not belong 
in $S_{\xi}$. Define $T \in \mathcal{L}(X)$ to be equal to 0 on $e_{\xi, n}$ for every $\xi$ and every $n \in F_{\xi}$. Also define $T$ to be the identity on the rest of the basis of $X$. Then the biorthogonal functional of the basis become a weak* basis for $X^{*}$ (see [9]), and $T^{*}$ is 0 on $e_{\xi, n}^{*}$ (the biorthogonal functional of $e_{\xi, n}$ ) for every $\xi$ and every $n \in F_{\xi}$. Also $T^{*}$ is identity on the rest of the weak* basis. Now let $\mathcal{A}$ denote the set of subsequences of $\left\{e_{\alpha}^{*}: \alpha \in D\right\}$ which are infinite branches of $D$ ( $e_{\alpha}^{*}$ denotes the biorthogonal functional of $e_{\alpha}^{*}$ for all $\left.\alpha \in D\right)$. This is a closed subset $\left(B a\left(X^{*}\right), \text { weak }{ }^{*}\right)^{\mathbb{N}}$ (this is because each "level" of $D$ has finitely many nodes thus finite many possible limits). Since $F_{\xi}$ is not in $S_{\xi}$ we have that $T \in \mathcal{A}-\mathcal{S C}_{\xi}(X, X)$ for all $\xi$. Thus Theorem 3.3 gives the existence of an infinite branch $b$ of $D$ and an infinite subset $N$ of $b$ such that for all finite subsets $F$ of $N$ we have that $\left.T^{*}\right|_{\operatorname{Span}}\left(e_{\alpha}\right)_{\alpha \in b \backslash F}$ is not an isomorphism.

\section{REFERENCES}

[1] Alspach, D.; Argyros, S.A., Complexity of weakly null sequences, Dissertationes Math. 321 (1992).

[2] Androulakis, G.; Dodos, P.; Sirotkin, G.; Troitsky, V., Classes of strictly singular operators and their products, to appear in Israel J. Math..

[3] Argyros, S.A.; Godefroy, G.; Rosenthal, H.P. Descriptive set theory and Banach spaces. Handbook of the geometry of Banach spaces, Vol. 2, 1007-1069, North-Holland, Amsterdam, (2003).

[4] Bossard, B. A coding of separable Banach spaces. Analytic and coanalytic families of Banach spaces. Fund. Math. 172 (2002), no. 2, 117-152.

[5] Diestel, J., personal communication.

[6] Farmaki, V., A Ramsey principle for Banach spaces implying the Gowers and Wagner dichotomies, preprint.

[7] Fonf, V.P., Boundedly complete basic sequences, $c_{0}$ subspaces and injections of Banach spaces, Israel J. Math. 89 (1995) no 1-3 173-188.

[8] Gowers, W.T. A Banach space not containing $c_{0}, l_{1}$ or a reflexive subspace. Trans. Amer. Math. Soc. 344 (1994), no. 1, 407-420.

[9] Johnson, W.B.; Rosenthal, H.P., On $w^{*}$-basic sequences and their applications to the study of Banach spaces, Studia Math. 18, (1972), 77-92.

[10] Kechris, A.S.,Classical Descriptive Set Theory, Grad. Texts in Math., 156, Springer-Verlag, 1995.

[11] Pelczynski, A., On strictly singular and strictly cosingular operators. I. Strictly singular and strictly cosingular operators in C(S) spaces, Bull. Acad. Polon. Sci. Sér. Sci. Math. Astronom. Phys. 13 1965 31-36.

[12] Rosenthal, H.P., On relatively disjoint families of measures, with some applications to Banach space theory. Studia Math. 37 (1970) 13-36.

[13] Tomczak-Jaegermann, N., Banach spaces of type p have arbitrarily distortable subspaces, GAFA 6 (1996) 1074-1082.

Department of Mathematics, University of South Carolina, Columbia, SC 29208, USA

E-mail address: giorgis@math.sc.edu

Department of Mathematics, Virginia Commonwealth University, Richmond, VA 23220, USA

E-mail address: kbeanland@vcu.edu 